\title{
The use of shape memory compression anastomosis clips in cholecystojejunostomy in pigs - a preliminary study
}

\author{
Piotr Holak ${ }^{1}$, Marek Jalynski ${ }^{1}$, Zdzisław Lekston $^{2}$, Izabella Babinska ${ }^{3}$, \\ Zbigniew Adamiak ${ }^{1}$
}

\author{
${ }^{1}$ University of Warmia and Mazury in Olsztyn, Faculty of Veterinary Medicine, \\ Department of Surgery and Rentgenology, Olsztyn, Poland \\ ${ }^{2}$ University of Silesia in Katowice, Faculty of Computer Science and Materials Science, \\ Department of Materials Science, Katowice, Poland \\ ${ }^{3}$ University of Warmia and Mazury in Olsztyn, Faculty of Veterinary Medicine, \\ Department of Pathophysiology, Forensic Veterinary Medicine and Administration, Olsztyn, Poland
}

Received February 23, 2015

Accepted November 10, 2015

\begin{abstract}
This paper reports on the use of compression anastomosis clips (CAC) in cholecystoenterostomy in an animal model. Cholecystojejunostomy was performed in 6 pigs using implants made of nickel-titanium alloy in the form of elliptical springs with two-way shape memory. The applied procedure led to the achievement of tight anastomosis with a minimal number of complications and positive results of histopathological evaluations of the anastomotic site. The results of the study indicate that shape memory NiTi clips are a promising surgical tool for cholecystoenterostomy in cats and dogs.
\end{abstract}

\section{CAC, cholecystoenterostomy, pigs, NiTi, biliary tract surgery}

Compression clips have been used in abdominal surgery, in particular in intestinal anastomosis, for over two centuries (Kaidar-Person et al. 2008), whereas clips made of nickel-titanium alloy have been introduced to surgical practice in the last decade (Holak et al. 2014). The use of shape memory compression clips in cholecystoenterostomy in animal subjects has not been described extensively in the literature. Extrahepatic biliary tract (EHBT) surgery is generally performed in dogs due to obstruction, traumatic injury $(7 \%)$ and cholecystitis $(5 \%)$. Biliary obstruction is caused by cholelithiasis, neoplasia (8\%), pancreatitis, inspissated bile, gallbladder mucoceles, parasites, duodenal foreign body and diaphragmatic hernia (Mehler et al. 2004). Cholecystoenterostomy is a method of choice in surgical treatment of the forementioned disorders. The aim of this study was to evaluate the usefulness of shape memory nickel-titanium clips for cholecystoenterostomy in animals.

\section{Materials and Methods}

The experiment was performed by the Department of Surgery and Roentgenology of the Faculty of Veterinary Medicine at the University of Warmia and Mazury in Olsztyn in collaboration with the Department of Materials Science at the University of Silesia in Katowice. It was approved under Resolution No. 12/2011 of the Local Ethics Committee for Animal Experimentation.

The experiment was carried out on 6 pigs of both sexes, same age and body weight of $25-28 \mathrm{~kg}$. Implants with two-way shape memory were used in cholecystojejunostomy in all animals. The implants were made of $\mathrm{Ni}_{50.8} \mathrm{Ti}_{49.2}$ and $\mathrm{Ti}_{50} \mathrm{Ni}_{48.7} \mathrm{Co}_{1,3}$ alloys in the form of elliptical double-coil rings, measuring $25 \mathrm{~mm} \times 7 \mathrm{~mm}$, with compressive strength of $7-10^{\prime} \mathrm{N}$. (Plate VIII, Fig. 1). Implants were cooled by immersion in liquid nitrogen for $15 \mathrm{~s}$. Cooled implants opened automatically to an angle of approximately $30^{\circ}$. When inserted, implants were locked under exposure to body heat.

Address for correspondence:

Piotr Holak

Department of Surgery and Rentgenology

Faculty of Veterinary Medicine

University of Warmia and Mazury in Olsztyn

Oczapowskiego 14, 10-718 Olsztyn, Poland

Phone +48 607927421

E-mail. piotr-holak@wp.pl

http://actavet.vfu.cz/ 
The animals were fasted for $12 \mathrm{~h}$ and prepared for surgery in accordance with standard anaesthesiology procedures for the species. Abdominal integuments were incised along the midline in the umbilical region. The gallbladder was punctured with a needle and bile was emptied with a syringe at the amount of $15-25 \mathrm{ml}$. The antimesenteric border of the jejunum was joined with the gallbladder wall with two stay sutures. Incisions were 5-7 mm-long, made through the jejunal and gallbladder walls. The gallbladder was incised in the fundus. Cooled and open clips were inserted through the incisions and locked under exposure to body heat, joining the walls of both organs (Plate VIII, Fig. 2). The walls in the lumina of both clipped organs were cut to guarantee patency. The 5-7 mm incisions in gallbladder and jejunal walls were closed with two layers of 3-0 absorbable sutures. The bile duct was tightly ligated using 3-0 nonabsorbable suture. Abdominal incisions were closed. The mean time of the surgical procedure was $40 \mathrm{~min}$. Analgesia was provided for 3 days, and antibiotics were administered for 7 days after the surgery. On the first and tenth day after the procedure, all animals were subjected to X-ray examinations of the abdominal cavity in lateral and sagittal planes with barium sulphate as the contrast medium. The animals were euthanised 14 days after surgery, and tissue samples were collected from the anastomotic site for histopathological analysis. Tissue samples were fixed in buffered formalin and embedded in paraffin blocks. Sections were subjected to HE and Masson's trichrome staining and immunohistochemical staining to determine the presence of the proliferating cell nuclear antigen (Dako, PCNA, clone PC10). The thickness of fused tissues was measured in the Nikon NIS-Elements application (10 measurements at randomly selected sites).

\section{Results}

Healing complications or lack of appetite were not observed in any of the animals after surgery. Contrast X-ray examinations performed on the first and tenth day after surgery did not reveal leaks or accumulation of the contrast medium in the gallbladder. Clips were expelled with faeces 6 to 9 days after the procedure. In one animal, clips were not found in faeces, and the time of excretion could not be determined. In one patient, the omentum was fused with the anastomotic site along a $25 \mathrm{~mm}$ section. In all animals, the gallbladder was completely emptied and reduced in width to $10-20 \mathrm{~mm}$ at the widest point (Plate IX, Fig. 3).

A macroscopic evaluation during histopathological analysis revealed that the anastomotic site was tight and patent (Plate IX, Fig. 4). The jejunal mucosa was swollen and somewhat hyperaemic. The lumen of the gallbladder contained minimal amounts of thick, greenorange bile without ingesta.

Microscopic evaluation of the anastomotic site was conducted on longitudinal sections. Bands of connective tissue running in different directions were observed, and most bands were positioned along the anastomotic site (Plate XI, Fig. 7). Numerous arterioles were noted, mostly under the gallbladder mucosa (Plate X, Fig. 5). The mean thickness of the anastomotic site, excluding the mucosa, was $1.049 \mathrm{~mm}$ ( $\min .0 .736 \mathrm{~mm}$, max. $1.361 \mathrm{~mm}$ ). Immunohistochemical evaluation revealed a low level of mitotic activity in fibrocytes. A weak PCNA-positive reaction was noted in approximately $50 \%$ of connective tissue cells at the anastomotic site (Plate X, Fig. 6). Loose connective tissue forming a continuous layer of tunica adventitia was the most visible structure.

Inflammatory changes were observed in the jejunal mucosa. The lamina propria was visibly swollen and infiltrated by mononuclear cells, including eosinophils, plasmocytes and lymphocytes. Numerous intraepithelial lymphocytes were noted between enterocytes. Intestinal villi were shorter and crypts were shallower. The most profound changes in the jejunal mucosa were observed at the point of direct contact with the gallbladder mucosa (Plate XI, Fig. 7). Mitotic activity in crypts was high, and strong PCNA-positive reactions were noted in approximately $80 \%$ of enterocytes.

The gallbladder mucosa was covered with a single layer of club-shaped epithelial cells, mostly bright cells, with numerous intraepithelial lymphocytes in between. At the point of contact with the jejunal mucosa, epithelial cells showed early symptoms of necrosis (acidophilic cytoplasm, loose nuclear chromatin). Swelling and infiltration by mononuclear cells, mostly lymphocytes, was noted under the epithelium. Lymphatic tissue was also composed of lymph follicles. Short and wide mucosal folds were supported by 
the connective tissue framework. Numerous mucous tubular glands with high levels of secretory activity were noted at the place of contact with the jejunal mucosa (Plate XI, Fig. 7). Tubular and follicular glands with lower levels of secretory activity were observed further from the anastomotic site.

\section{Discussion}

The absence of publications discussing the usefulness of shape memory Ni-Ti compression anastomosis clips in cholecystojejunostomy in veterinary literature prompted the authors to conduct an experiment on a porcine model. The surgical procedure was performed with the use of two-way shape memory clips which, in the authors' opinion, are easier to use than clips with one-way shape memory because they shorten the time of the surgical procedure and do not have to be manually opened with a special tool. The unquestioned advantage of two-way shape memory clips is that they are expelled naturally with faeces (Nudelman et al. 2002)

The width of incisions at the anastomotic site approximated the size of the clip, and it was not reduced, as it may be the case when manual sutures are applied. The width of the actual anastomosis can be thus programmed subject to need (the animal's size) by using clips of appropriate size. According to the authors, when applied evenly across the entire clip, compressive strength of 7-10 $\mathrm{N}$ is optimal for creating tight cholecystojejunal anastomosis, and it contributes to necrosis, which causes the clip to be excreted from the body. Minimal quantities of suture materials were used to close incisions in jejunal and gallbladder walls, thus minimizing the risk of leakage that is sometimes observed in hand-sewn anastomoses. In one patient, omental adhesion was noted after cholecystojejunostomy. Histopathological analysis did not reveal significant differences between that individual and the remaining subjects. Differences in histopathological results could suggest that omental adhesion was caused by a leak around the anastomosis.

X-ray examination with a contrast medium was performed on the first day after surgery to determine possible leaks. The second X-ray exam, performed 10 days after the procedure when clips had been expelled by all animals, revealed tight cholecystojejunal anastomosis. Presence of the contrast medium or ingesta was not observed in the gallbladder during $\mathrm{X}$-ray exams. Tensile strength is a critical factor in anastomosis because leaking ingesta and bile can lead to peritonitis (Peddle et al. 2008).

A histological evaluation of cholecystojejunal anastomosis confirmed tight fusion of the connective tissue. Changes in the jejunal mucosa in the anastomotic site could be attributed to the irritant effects of leaking bile. The anastomosis should be examined after a longer period of time to determine the type of morphological changes in the jejunal and gallbladder mucosa and to describe the capability for repair of the jejunal mucosa.

The use of two-way shape memory NiTi clips in cholecystojejunostomy delivered promising results in a porcine model. Those findings will be used in successive experiments with prolonged time of postoperative histopathological evaluation to determine the character of bile-induced changes in the jejunal mucosa (Amsellem et al. 2006). In clinical patients (cats and dogs), cholecystoenterostomy is usually the only form of treatment during surgical interventions (Tangner 1984). In those cases, NiTi clips with shape memory could be used to create tight and reliable cholecystojejunal anastomoses. NiTi clips are relatively inexpensive, which is a very important consideration in veterinary practice.

\section{References}

Amsellem PM, Seim HB, MacPhail CM, Bright RM, Twedt DC, Wrigley RH, Monnet E 2006: Long-term survival and risk factors associated with biliary surgery in dogs: 34 cases (1994-2004). J Am Vet Med Assoc 229: $1451-1457$ 
Holak P, Jalynski M, Adamiak Z, Lekston Z, Morawiec H, Otrocka-Domagala I, Przyborowska P, Pazdzior K 2014: The use of shape memory NiTi alloy clips in small bowel anastomosis in pigs. Vet Med-Czech 59: 124-128

Kaidar-Person O, Rosenthal RJ, Wexner SD, Szomstein S, Person B 2008: Compression anastomosis: history and clinical considerations. Am J Surg 195: 818-826

Mehler SJ, Mayhew PD, Drobatz KJ, Holt DE 2004: Variables Associated with Outcome in Dogs Undergoing Extrahepatic Biliary Surgery: 60 Cases (1988-2002). Vet Surg 33: 644-649

Nudelman IL, Fuko V, Greif F, Lelcuk S 2002: Colonic anastomosis with the nickel-titanium temperaturedependent memory-shape device. Am J Surg 183: 697-701

Peddle GD, Carberry CA, Goggin JM 2008: Hemorrhagic bile pleuritis and peritonitis secondary to traumatic common bile duct rupture, diaphragmatic tear, and rupture of the spleen in a dog. J Vet Emerg Crit Care 18: 631-638

Tangner CH 1984: Cholecystoduodenostomy in the dog. Comparison of two techniques. Vet Surg 13: 126-134 
Plate VIII

Holak P. et al.: The use of shape ... pp. 403-406

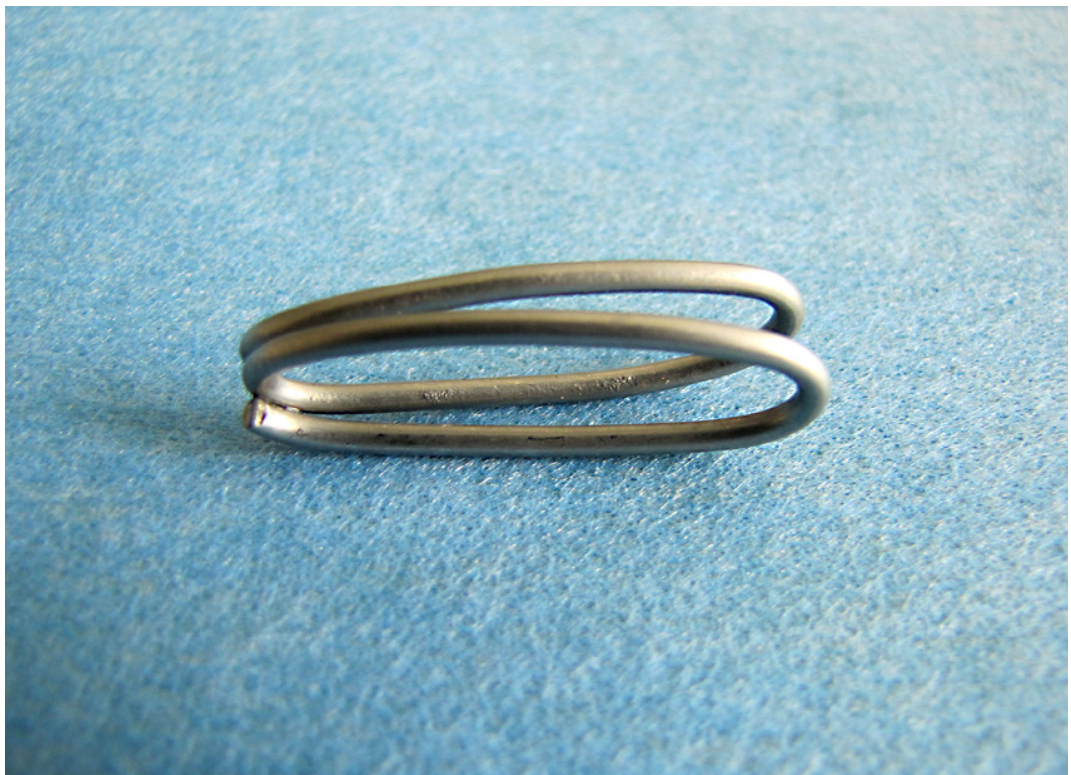

Fig. 1. A two-way shape memory NiTi clip at room temperature

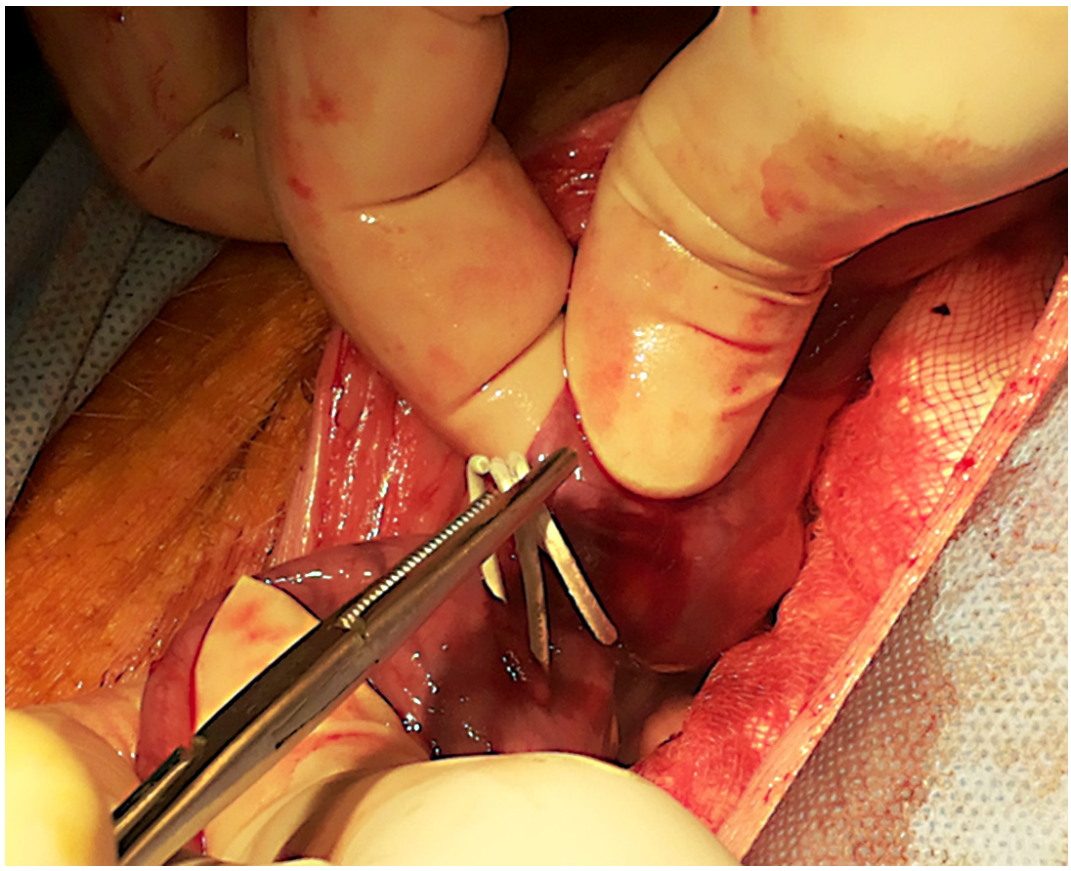

Fig. 2. Insertion of a clip into the gallbladder and the jejunum. The clip opens automatically when cooled 
Plate IX

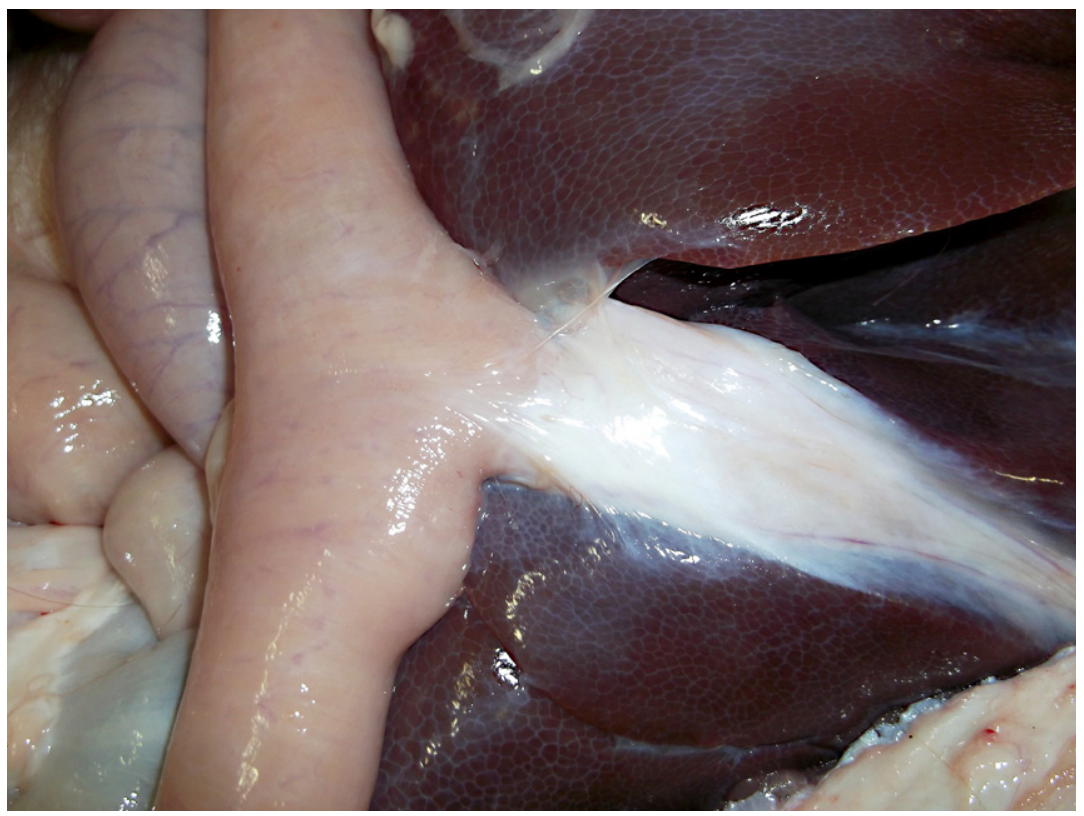

Fig. 3. Cholecystojejunostomy - autopsy image

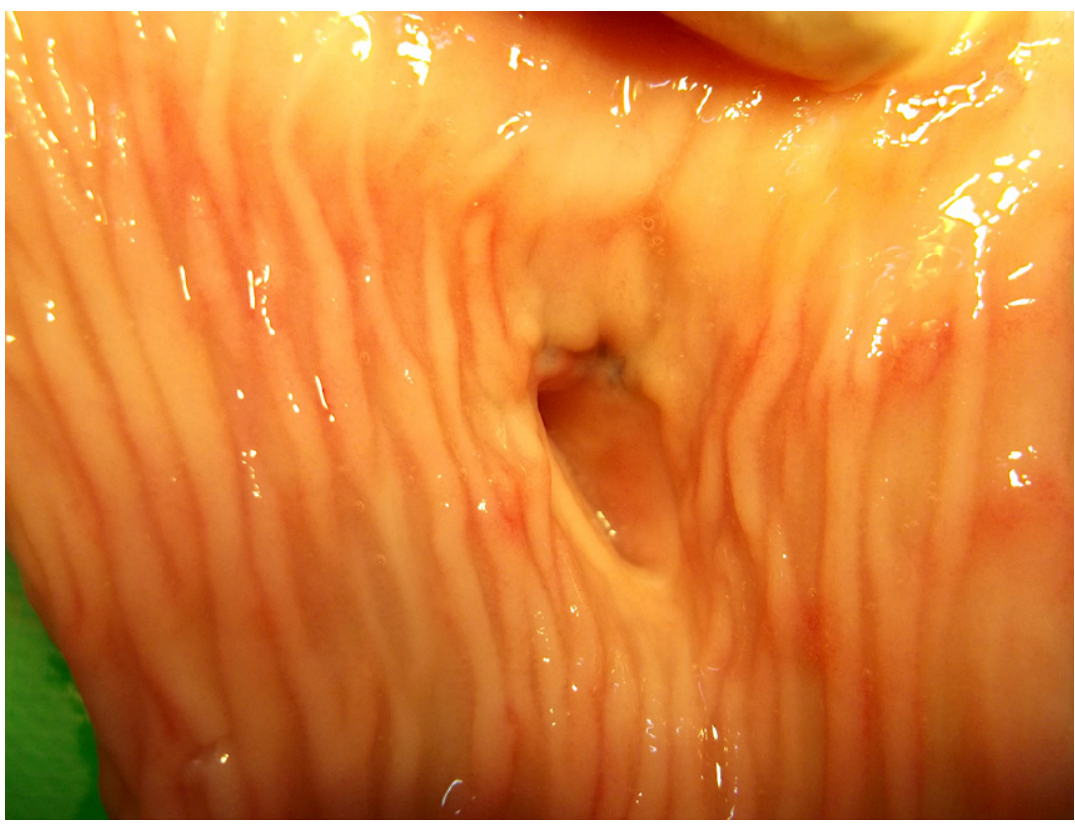

Fig. 4. Cholecystojejunostomy - view from the intestinal lumen 


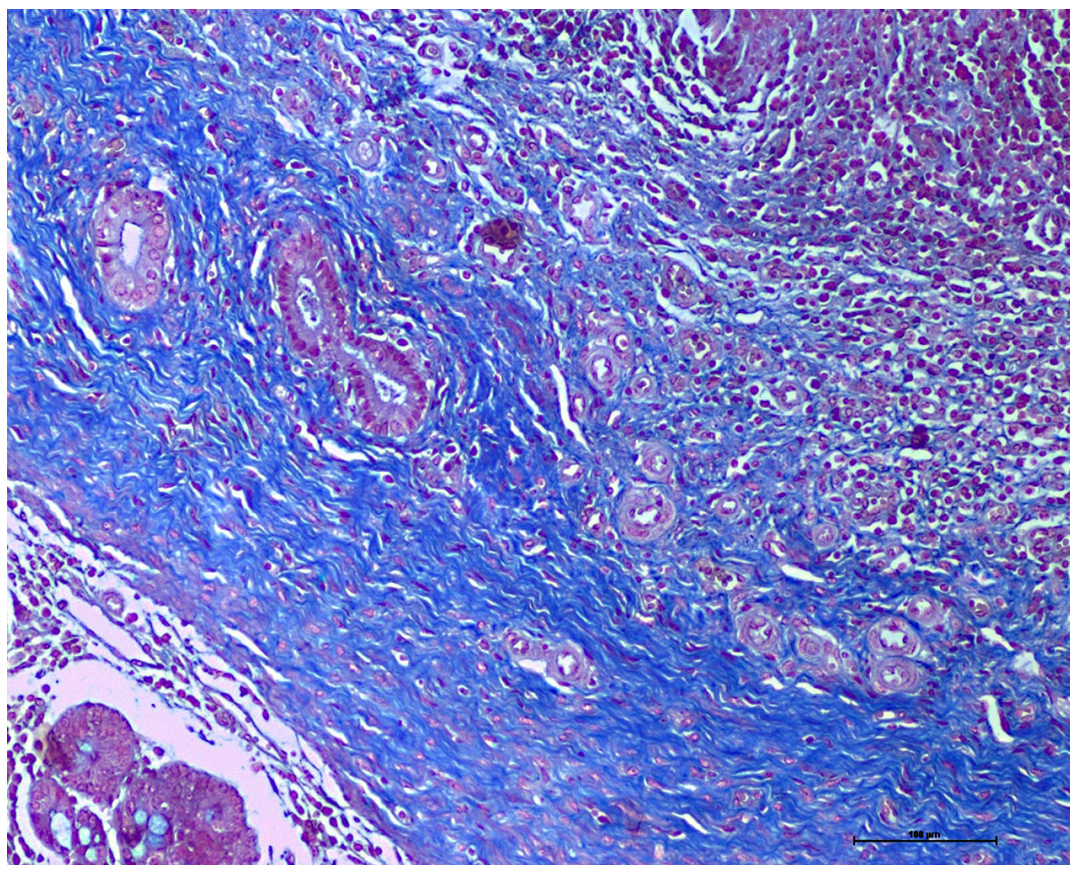

Fig. 5. Fused connective tissue between gallbladder and jejunal walls with numerous arterioles. Masson's trichrome stain, magnification $\times 10$

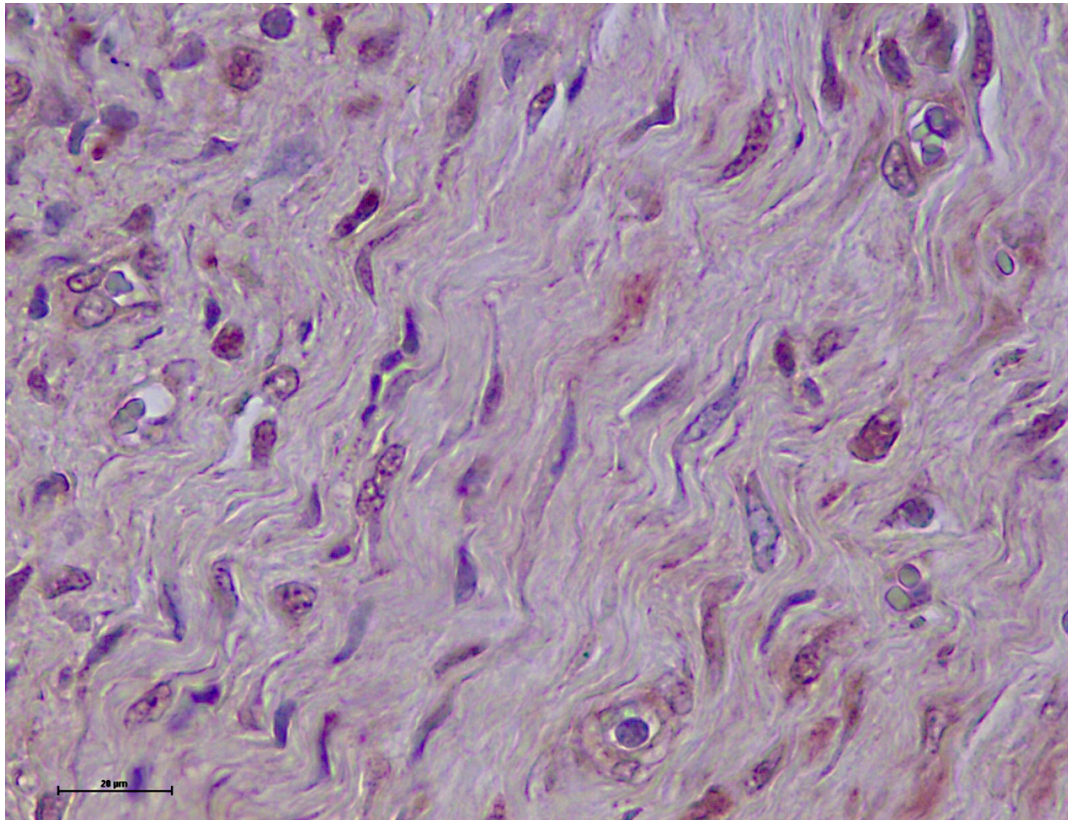

Fig. 6. Weak PCNA-positive reaction in the cell nuclei of connective tissue forming cholecystojejunal anastomosis, magnification $\times 40$ 


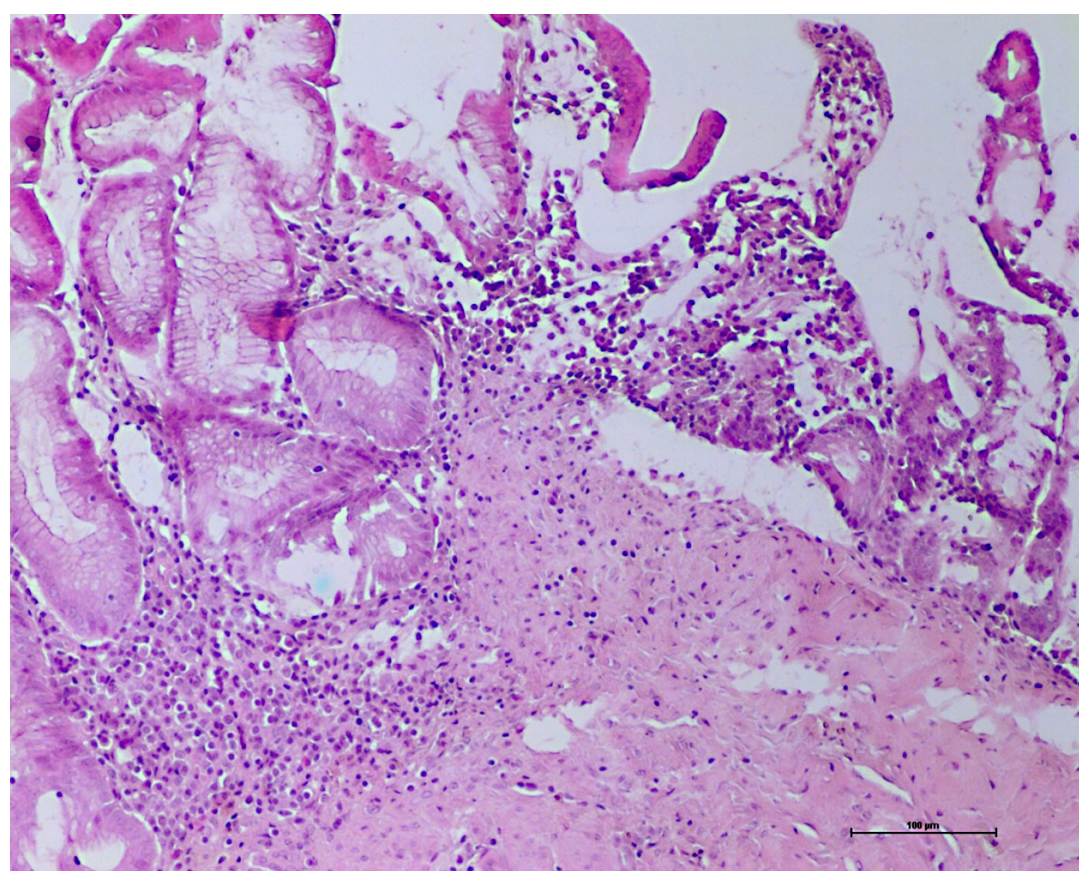

Fig. 7. Area of direct contact between the jejunal mucosa and the gallbladder mucosa. HE stain, magnification $\times 10$ 